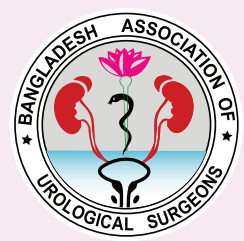

\title{
Miniaturization of PCNL; Necessity or What?
}

\author{
Mofizur Rahman', Mohammed Monowar-Ul-Hoque ${ }^{2}$, Kazi Mohammad Monwarul Karim³, \\ AKM Akramul Bari ${ }^{4}$, Md Asaduzzaman Asad ${ }^{5}$, Md. Tanvir Rahman ${ }^{6}$
}

\author{
Received: $01-06-2020$ \\ Accepted: 09 - 09 - 2020 \\ Conflicts of interest: None
}

Keywords: Percutaneous nephrolithotomy (PCNL), minimally invasive PCNL (Mini-PCNL), standard PCNL

\begin{abstract}
Objective: Now a days, percutaneous nephrolithotomy (PCNL) is the treatment of choice for retrieval of renal and proximal ureteral calculi. The primary goal of PCNL is to achieve stone free status while minimizing morbidity and complications. In recent years, the instruments used have been miniaturized in an effort to decrease morbidity associated with standard PCNL as well as increase the efficacy of stone removal. The aim of this study was to compare the safety and efficacy of PCNL using different tract size.
\end{abstract}

Patients and Methods: This hospital based prospective interventional study was conducted on patients with 1 to $4 \mathrm{~cm}$ renal stones who underwent PCNL either by Mini or by Standard PCNL technique in Chattogram Medical College Hospital and different private hospitals in Chattogram from July 2016 to October 2018. Patients aged above 12 years of age, irrespective of gender with normal renal function were evaluated to compare stone clearance, access time, fluoroscopy time for access, total operative time, need for blood transfusion, postoperative hospital stay, postoperative pain, fever, urinary leakage and other complications between two groups. Those who had previous history of open renal surgery, active urinary tract infection, renal malformation, uncorrected coagulopathy and morbid obesity were excluded.

Results: A total of 78 patients were enrolled consecutively for PCNL who were divided equally into two groups randomly for minimally invasive PCNL (Mini-PCNL) and Standard $P C N L$. The average stone size in mini-PCNL group was $2.59 \pm 0.89 \mathrm{~cm}$, and $2.66 \pm 0.97 \mathrm{~cm}$ in standard-PCNL group ( $p=0.7)$. Mean tract size was $18.53 \pm 1.29 \mathrm{~F}(16-20)$ and $26.11 \pm$ $4.61 F$ (24-30) respectively with P value Elt;0.001. In mini-PCNL operative time was significantly longer than that of standard PCNL with $112.11 \pm 20.29$ vs. $98.68 \pm 19.75$ minutes respectively with $p=.004$. Conversely, there was an advantage of mini-PCNL over the standard one in terms of a significantly reduced hemoglobin drop $(0.5 \pm 0.26$ vs. $0.83 \pm$ $0.32 \mathrm{gm} \%, p$ value 0.001$)$ and hospital stay $(2.18 \pm 0.77$ vs. $3.39 \pm 1.10$ days, $p$ value $=$ 0.001), respectively though there was no statistical difference in terms of stone clearance rates between two groups ( $86.84 \%$ vs. $92.11 \%, p=0.45)$. There was no statistical difference in terms of visual analogue scale (VAS) score (5.55 \pm 1.54 vs. 6.24 \pm 1.6$)$ for pain perception. The complication rate of mini-PCNL had no significant difference with that of standard PCNL (10.52\% vs $12.50 \%, p=0.72)$. No statistical difference was recorded in terms of postoperative fever (eŠ38C) between two groups (2 in each group, 5.2\%, $p=1$ Blood transfusion requirement was much less in mini PCNL group $(10.52 \%$ vs. $34.2 \%, p=0.01)$.

Conclusion: In addition to minimal bleeding and excellent stone clearance, mini- PCNL has several features for which it should be considered as an alternative or adjunct tostandard PCNL, URS, and ESWL. These include safe supra-costal puncture, excellent access to nearly all calyces and upper ureter, less hospital stay and suitable for large stones also. Future studies should continue to refine methods to assess complexity and safety and to determine consensus on the use of mini- PCNL.

1. Assistant Professor, Department of Urology, Chattogram Medical College, Chattogram

2. Associate Professor, Department of Urology, Chattogram Medical College, Chattogram

3. Associate Professor, Department of Urology, Chattogram Medical College, Chattogram

4. Assistant Registrar, Department of Urology, National Institute of Kidney Diseases \& Urology, Dhaka

5. Urology Phase-B Student, Chattogram Medical College, Chattogram

6. MS (Urology) Thesis Part Student, Chattogram Medical College, Chattogram

Correspondence to: Dr. Mofizur Rahman, Assistant Professor, Chattogram Medical College, Chattogram, Email: uromofiz@yahoo.com 


\section{Introduction:}

Amongst the disease of urinary tract renal stone is the third most common. Most incidence of stone occurrence is the fourth to sixth decades of life. Male are affected two to three times more often than female. Stone formation has migrated from the lower to the upper urinary tract with westernization of global culture and the disease is getting increasingly gender blind. ${ }^{1}$ Over the last two decades, open surgery have replaced by Percutaneous Nephrolithotomy (PCNL) and Extracorporeal Shock Wave Lithotripsy (ESWL) for the management of kidney stones. ${ }^{2}$ With the use of invasive and noninvasive techniques, open surgery has been used only at a rate of $1-2 \%$ in the management of renal stones. ${ }^{3}$ Fernström and Johansson described PCNL for the first time in $1976 .{ }^{4}$ PCNL has been recommended as the therapy of choice for large renal calculi $(>20 \mathrm{~mm})$ and also for smaller stones $(10-20 \mathrm{~mm})$ of the lower renal pole when unfavorable factors for ESWL exist according to the updated European Association of Urology (EAU) guidelines. ${ }^{5}$ Excellent stone-free rates (SFR) following PCNL have been reported, which range from $76 \%$ to $98 \% .{ }^{6}$ However, PCNL is still a challenging surgical technique and can be associated with significant complications that may compromise its efficacy. In order to decrease morbidity associated with larger instruments like blood loss, postoperative pain and potential renal damage, a modification of the technique of standard PCNL has been developed. This is performed with a miniature endoscope via a small percutaneous tract (11-20 F) and was named as minimally invasive PCNL or mini-PCNL or mini-Perc.This technique was first developed and accomplished by Jackman et al. in the pediatric population with the use of an $11 \mathrm{~F}$ access tract. ${ }^{7}$ Since then, the method has become a treatment option for adults as well. ${ }^{8,9}$ The primary goal of PCNL is to achieve stone-free status while minimizing morbidity and complications. Usually, the term miniPCNL is used for access sheaths $20 \mathrm{Fr}$ or below $20 \mathrm{Fr}$. However, the terminology has not been standardized yet and the procedure lacks a clear definition. ${ }^{10}$ Access sizes ranging from $11 \mathrm{~F}$ to $20 \mathrm{~F}$ have been reported in the some literature ${ }^{11}$ whereas 14 to $20 \mathrm{~F}$ has been described in some other papers. ${ }^{12}$ The mini-perc technique is believed to have several advantages, including decreased blood loss, increased maneuverability, less pain and shorter hospital stays. As the risk for bleeding complications is related to the number and caliber of tracts used ${ }^{13}$, limited transfusion rates have been reported with this technique. ${ }^{14}$ However, reducing tract size may adversely affect some procedure related factors. Although several studies have shown the safety and efûcacy of miniPCNL for small calculi, the fear of reduced visibility, a prolonged operating time, and lower primary stonefree rates owing to the reduced shaft diameter has resulted in reluctance to apply miniaturized instruments in patients with a larger stone burden. ${ }^{15}$ Thus, each of the types of PCNL has its own advantages and disadvantages and has been selected by different surgeons. Moreover, the choice also depends on surgeons experience and expertise. In Bangladesh, due to recent advancement of endourology, PCNL is frequently done for retrieval of renal stone and one of the fundamental steps of PCNL is the creation of the nephrostomy access. There is lack of data comparing different techniques in our context. Therefore, it was necessary to conduct further research to evaluate the efficacy and safety of mini-PCNL. Therefore, we considered it is necessary to perform a prospective interventional study in our setting comparing the efficacy and safety of the mini-percutaneous nephrolithotomy in comparison to standard PCNL. The aim of this study was to demonstrate that minipercutaneous nephrolithotomy might represent a reasonable procedure in patients with smaller stones offering a similar outcome as standard percutaneous nephrolithotomy with advantage of reduced morbidity. Our objectives were to evaluate the efficacy and safety to compare the stone clearance rate to observe the access time, fluoroscopy time for access and total operative timeto compare the postoperative pain between two groups, to evaluate any per operative or immediate post-operative complications like excessive bleeding, peri-operative transfusion requirements, post-operative fever and urinary leakage,post-operative hospital staybetween two groups.

\section{Methods}

This was a randomized controlled study done in the Urology Department, Chattogram Medical College Hospital and different private hospitals of Chattogram, Bangladesh from July 2016 to June 2018,based on patients admitted with renal calculi and underwent PCNL during the study period.Patients was selected by consecutive sampling method considering the inclusion and exclusion criteria \& divided randomly into two groups using an online based statistical 
calculator (Graph Pad Software, Inc.2017; https:// www.graphpad.com/quickcalcs/randomize1.cfm). Group A for the miniaturized PCNL technique and Group B for the conventional technique.To estimate the sample size for hypothesis testing, standard statistical formula was used.Patients undergoing PCNL for renal stone, where patients age 12 years or more, stone size $1 \mathrm{~cm}$ to $4 \mathrm{~cm}$, with normal renal function were evaluated for study.Those who had previous history of open renal surgery, active UTI, renal malformation, uncorrected coagulopathy \& morbid obesity were excluded.

After proper counseling and detailed explanation of procedure, obtaining written informed consent, all patients were evaluated by detailed history, thorough physical examinations and relevant investigations. Negative urine culture were ensured before surgical intervention. Co-morbidities were addressed and controlled preoperatively.

All patients underwent PCNL under general anesthesia and received intravenous broad spectrum antibiotics prior to manipulation. Initially, on lithotomy position, a $6 \mathrm{Fr}$ ureteric catheter was placed transurethrally over the 0.035 inch guide wire under fluoroscopic guidance. Then patient was placed at prone position. Pelvi-calyceal system of the targeted while kidney was opacified with diluted contrast media under fluoroscopy. Initial puncture was decided on retrograde pyelogram findings. Percutaneous access was created using an $18 \mathrm{G}$ access needle into the selected calyx. A guidewire was placed into the collecting system. The nephrostomy tract was dilated by gradual dilatation technique with Metallic Alkan Dilator (Karl Storz) 16 to $20 \mathrm{Fr}$ for mini PCNL and 24 to 28 Fr for standard PCNL. Then Amplatz sheath (Cook / Rusch Medical) was positioned into the renal collecting system accordingly. The stone was disintegrated using pneumatic lithotripter. Nephroscope with forceps was used to retrieve stones from calyces. Once complete clearance was confirmed fluoroscopically and endoscopically, a 5/6 F JJ stent was placed antegradely. On completing the procedure, the Amplatz sheath was removed after keeping a nephrostomy tube in situ.
On postoperative day 1, a plain X-ray was obtained to document stone clearance. Nephrostomy tube was removed if the urine was not hemorrhagic and stone clearance was achieved. The Foley's catheter was removed on $2^{\text {nd }}$ postoperative day. The JJ stent was removed after 4 weeks. Re-PCNL, URS (Ureterorenoscopy) and ESWL was considered as accessory treatment alternatives when indicated. Close follow up and recording was done to search for any complications encountered immediately after operation. $\mathrm{Hb} \%$ will be estimated on $1^{\text {st }}$ post-operative day. Post-operative pain was measured using "Visceral analogue Score (VAS)". Any need for blood transfusion was recorded. Patients were discharged with appropriate follow up advices if no complication arises.

\section{Result:}

A total of 78 patients were enrolled consecutively for PCNL who were divided equally into two groups randomlyfor minimally invasive PCNL (Mini-PCNL) and Standard PCNL. The average stone size in miniPCNL group was $2.59 \pm 0.89 \mathrm{~cm}$, and $2.66 \pm 0.97 \mathrm{~cm}$ in standard-PCNL group $(\mathrm{p}=0.7)$. Mean tract size was $18.53 \pm 1.29 \mathrm{~F}(16-20)$ and $26.11 \pm 4.61 \mathrm{~F}(24-$ 30)respectively with $P$ value \&lt;0.001. In mini-PCNL operative time was significantly longer than that of standard PCNL with112.11 \pm 20.29 vs. $98.68 \pm 19.75$ minutes respectively with $p=.004$. Conversely, there was an advantage of mini-PCNL overthe standard one in terms of a significantly reduced hemoglobin drop $(0.5 \pm 0.26$ vs. $0.83 \pm 0.32$ gram $\%, P=0.001)$ andhospital stay $(2.18 \pm 0.77$ vs. $3.39 \pm 1.10$ days, $\mathrm{P}=0.001)$, respectively though there was no statistical difference in terms ofstone clearance rates between two groups ( $86.84 \%$ vs. $92.11 \%, \mathrm{p}=0.45)$. There was no statistical difference in terms ofvisual analogue scale (VAS) score (5.55 \pm 1.54 vs. $6.24 \pm 1.6)$ for pain perception. The complication rate of mini-PCNL had nosignificant difference with that of standard PCNL $(10.52 \%$ vs $12.50 \%, p=0.72$ ). No statistical difference was recorded interms of postoperative fever ( $\left.\mathrm{e}^{\prime \prime} 38 \mathrm{C}\right)$ between two groups ( 2 in each group, $5.2 \%$, $p=1$ Blood transfusion requirement wasmuch less in mini PCNL group (10.52\% vs. $34.2 \%, p=0.01)$.

Table I : Demographics of patients in the mini-PCNLE conventional percutaneous nephrolithotomy groups (mean \pm SD or \%)

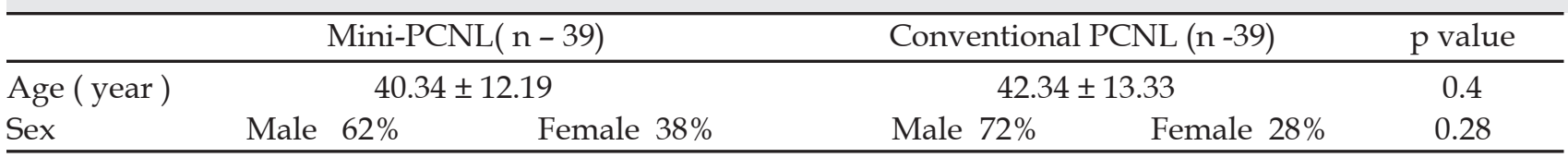


Table II : Surgical parameters in the mini-PCNLE conventional percutaneous nephrolithotomy groups (mean \pm SD or \%)

\begin{tabular}{lccc}
\hline & Mini-PCNL group A & Standard-PCNL group B & P value \\
\hline Number of patients & 39 & 39 & \\
Stone size & $2.59 \pm 0.89$ & $2.66 \pm 0.97$ & 0.7 \\
Mean Tract size & $18.32 \pm 1.29$ & $26.11 \pm 4.61$ & $<0.001$ \\
Operative time & $112 \pm 20.29$ & $98.68 \pm 19.75$ & 0.004 \\
Hemoglobin drop & $0.5 \pm 0.26$ & $0.83 \pm 0.32$ & $<0.001$ \\
Hospital stay & $2.18 \pm 0.77$ & $3.39 \pm 1.10$ & $<0.001$ \\
Stone clearance rate & 86.84 & 92.11 & 0.45 \\
Visual analogue scale & $5.55 \pm 1.54$ & $6.24 \pm 1.6$ & \\
Complication rate & 10.52 & 12.50 & 0.72 \\
Postoperative fever & 5.2 & 5.2 & 1 \\
Bloodtransfusion requirement & 10.52 & 34.2 & 0.01 \\
\hline
\end{tabular}

\section{Discussion}

The "mini-perc" or mini-PCNL technique was first developed for children and reported in 1997 by Jackman et al. ${ }^{16}$ and Helal et al. ${ }^{17}$ The advantages of the "mini-perc" technique highlighted by these authors included decreased renal and body wall trauma, adjustable length, and convenient working size for pediatric instruments.

Even in the first series regarding mini-PCNL, the stone free rate (SFR) was high enough although the stone burden was relatively low. Jackman et $\mathrm{a}^{16}$ reported an SFR of $85 \%$ in children and $89 \%$ in adults with a stone burden $1.2 \mathrm{~cm}^{2}$ and $1.5 \mathrm{~cm}[2]$, respectively. Similar SFRs between mini-PCNL and PCNL have been reported by most authors except Giusti et al.[18] who reported lower SFRs despite longer operative times.Similar to the pediatric experience, early stonefree success with the adult "mini-perc" was equivalent to that of recent series. Saxby et al. ${ }^{19}$ and Lingeman ${ }^{20}$ have reported stone-free rates of $85 \%$ and $95 \%$, respectively, for stones measuring $2.0 \mathrm{~cm}$ or less in size. This is consistent with the $86.84 \%$ stone-free rate of the current series. Additionally, in the kidney in which a small residual fragment was seen 1 week postoperatively there is a reasonable chance of spontaneous passage.

Jackman SV (1998) ${ }^{14}$ has mentioned limited blood loss, increased maneuverability, decreased postoperative pain \& limited hospital stay in cases of mini-PCNL. Limitations of the procedure include the necessity to disintegrate stones into small enough fragments to fit through a reduced sizedsheath which results in longer operative times.

Cheng et al. (2010) ${ }^{21}$ stated no significant differences in SFR between the mini-PCNL and PCNL. However, a better stone clearance rate was demonstrated for multiple calyceal stones when mini-PCNL was performed (85.2\% vs. 70\%). Zhong W et al. 2011[22] stated Higher SFR was achieved in the treatment of staghorn stones with mini-PCNL and the creation of multiple access tracts $(89.7 \%$ vs. $68 \%)$.

Giusti et al. (2007)[23] \& Mishra et al. (2011) ${ }^{24}$ has found longer operative times associated with mini-PCNL (155.5 $\mathrm{min}$ and $45 \mathrm{~min}$ vs. $106.6 \mathrm{~min}$ and $31 \mathrm{~min}$ respectively). Abdelhafez MF (2013)[25] stated MiniPCNLas more effective when treating smaller $(<20$ $\mathrm{mm}$ ) rather than larger $(>20 \mathrm{~mm})$ renal stones (SFR $90.8 \%$ vs. $76.3 \%$ ). In current series operating time $112.11 \pm 20.29$ minwere significantly larger than standard PCNL (98.68 $\pm 19.75 \mathrm{~min})$, which is very similar with those previous study.

Traxer et al. (2001) $)^{16}$ measured and compared the extent of renal parenchyma injury also concluded that renal parenchyma damage resulting from the creation of a nephrostomy tract is small compared to overall renal volume regardless of the size of the nephrostomy tract. However, the benefit of mini-PCNL remains as the use of smaller access sheaths resulted in reduced intraoperative blood loss, less postoperative pain and shorter hospital stay. Cheng F et al. (2010) ${ }^{21} \&$ Mishra et al. $(2011)^{24}$ stated an advantage of mini-PCNL over the conventional procedurein terms of a significantly 
reduced hemoglobin drop $(0.53 \mathrm{~g} / \mathrm{dl}$ and $0.8 \mathrm{~g} / \mathrm{dl}$ vs. $0.97 \mathrm{~g} / \mathrm{dl}$ and $1.3 \mathrm{~g} / \mathrm{dl}$ respectively and the need for blood transfusion $(1.4 \%$ vs. $10.4 \%)$. Mishra $S$ et al. $(2011)^{24}$ concluded that analgesic requirement has also been found significantly decreased in mini-PCNL when compared to standard PCNL (55.4 g vs. $70.2 \mathrm{~g}$ tramadol). Knoll et al. (2010)27 \& Mishra et al.(2011)[24] stated that hospital stay was significantly shorter after mini-PCNL (3.8 days and 3.2 days vs. 6.9 days and 4.8 days respectively).In our series Hospital stay was also significantly shorter after mini-PCNL $(2.18 \pm 0.77$ v 3.39 \pm 1.10 days), less post operative pain were also noted (Visual analogue scale: $5.55 \pm 1.54$ v $6.24 \pm 1.6$ ), diminished $\mathrm{Hb} \%$ drops $(0.5 \pm 0.26 \mathrm{gm} \%$ v $0.83 \pm 0.32$ gm \%), less blood transfusion(10.52\% v $34.2 \%)$.

In conclusion, the technique and rational for performing a "mini-perc" in selected patients are presented in this report. Early experience suggests that its stone clearance rate is comparable with that of a standard PCNL and that it may have advantages over the current procedure. These potential advantages include decreases in blood loss, transfusion rate, pain, and length of hospital stay. More extensive studies are needed to validate these hypotheses. It is likely that this technique will become an important alternative in the endourologist's armamentarium for the quick and definitive treatment of smaller stones in selected patients.

\section{References:}

1. Pearle, MS, Antonelli, JA \&Lotan, Y 2016, 'Urinary Lithiasis: Etiology, Epidemiology, and Pathogenesis' in Wein, AJ, Kavoussi, LR. Partin, AW \& Peters CA, (eds), Campbell-Walsh Urology, $11^{\text {th }}$ edn, pp. 1188-1199, Elsevier, Philadelphia.

2. Segura JW, Preminger GM, Assimos DG, Dretler SP, Kahn RI, Lingeman JE, Macaluso JN Jr, McCullough DL. Nephrolithiasis Clinical Guidelines Panel Summery Report on The Management of Staghorn calculi: The American Urological Association Nephrolithiasis Clinical Guidelines Panel. J Urol 1994;151:1648-1651.

3. Stoller, ML 2013, 'Urinary Stone Disease', in McAninch, JW \&Lue TF, (eds), Smith ETanagho's General Urology, $18^{\text {th }}$ edn, pp. 249-279, McGrawHill, California.

4. Arslan B, Akbulut MF, Onuk O, Küçüktopçu O, Çilesiz NC \& Ozkan A et al. 2017, 'A comparison of Amplatz dilators and metal dilators for tract dilatation in mini-percutaneous nephrolithotomy', Int Urol Nephrol, vol. 49, pp. 581-585.

5. Türk C, Knoll T, Petrik A, Sarica K, Skolarikos A, Straub M, Seitz C 2016, 'EUA Guidelines on Urolithiasis', Arnhem (The European Association of Urology (EAU).

6. Michel, MS, Trojan, L \&Rassweiler, JJ 2007, 'Complications in Percutaneous Nephrolithotomy', EurUrol,vol.51,no.4,pp.899906.

7. Jackman, SV, Hedican, SP, Peters CA and Docimo, SG 1998, ' Percutaneousnephrolithotomy in infants and pre-school age children: Experience with a new technique', World J Urol, vol. 52, pp. 697701.

8. Chan, DY and Jarrett, TW 2000, 'Minipercutaneous nephrolithotomy', J Endourol, vol. 14, pp. 269-72.

9. Lahme S, Bichler KH, Strohmaier WL, Götz T 2001, 'Minimally invasive PCNL in patients with renal pelvic and calyceal stones', Eur Urol, vol. 40,pp. 619-24.

10. Ferakis, N and Stravroppoulos, M 2015, 'Mini percutaneous nephrolithotomy in the treatment of renal and upper ureteric stones: Lessons learned from a review of literature', Uro annals, vol. 7, pp. 141-148.

11. Li, X, He, Z, Wu, K, Li, SK,Zeng, G, Yuan, J, et al. 2009, 'Chinese minimally invasive percutaneous nephrolithotomy:The Guangzhou experience', J Endourol, vol. 23, pp. 693-7.

12. Wright, A, Rukin, N, Smith, D, Rosette, J and Somani, BK 2016, 'Mini, ultra, micro' nomenclature and cost of these new minimally invasive percutaneous nephrolithotomy (PCNL) techniques', The AdvUrol, Vol. 8(2), pp.142-146.

13. Losty, P, Surana, R and O'Donnell, B 1993, 'Limitations of extracorporeal shock wave lithotripsy for urinary tract calculi in young children', J PedSurg, vol. 28, pp. 1037-9.

14. Jackman, SV, Docimo, SG, Cadeddu, JA, Bishoff, JT, Kavoussi, LR and Jarrett TW 1998, 'The "miniperc" technique: a less invasive alternative to percutaneous nephrolithotomy' ,World J Urol, vol.16, pp. 371-4. 
15. Nagele, U, Anastasiadis, AG, Schilling, DA, et al. 2007, 'Introducing a new sealant applicator for easy, safe, and quick closure of a minipercutaneous nephrolitholapaxy access tract', JEndourol, vol. 21, pp. 393-396.

16. Jackman SV, Hedican SP, Docimo SG, Peters CA (1997) Miniaturized access for pediatric percutaneous nephrolithotomy. J Endourol 11: S133.

17. Helal M, Black T, Lockhart J, Figueroa TE 1997 The Hickman peel-away sheath: alternative for pediatric percutaneousnephrolithotomy. J Endourol 11: 171 \pm 172 .

19. Saxby MF, Sorahan T, Slaney P, Coppinger SWV 1997 Acase-control study of percutaneous nephrolithotomy versus extracorporeal shock wave lithotripsy. Br J Urol 79: 317 \pm 323 .

20. Lingeman JE, Coury TA, Newman DM, Kahnoski RJ, Mertz JHO, Mosbaugh PG, Steele RE, Woods JR 1987 Comparison of results and morbidity of percutaneous nephrostolithotomy and extracorporeal shock wave lithotripsy. J Urol 138:485 \pm 490 .

21. Cheng F, Yu W, Zhang X, Yang S, Xia Y, Ruan Y.2010 Minimally invasive tract in percutaneous nephrolithotomy for renal stones. J Endouro;24:1579 82.
22. Zhong W, ZengG,WuW,ChenW,WuK.2011 Minimally invasive percutaneous nephrolithotomy with multiple mini tracts in a single session in treating Staghorn calculi.UrolRes;39:117 22.

23. Giusti G, Piccinelli A, Taverna G, Benetti A, Pasini L, Corinti M, et al. Miniperc? No, thank you! EurUrol 2007;51:810 4.

24. Mishra S, Sharma R, Garg C, Kurien A, Sabnis R, Desai M. 2011Prospective comparative study of mini perc and standard PNL for treatment of 1 to 2 cm size renal stone. BJU Int;108:896 9.

25. Abdelhafez MF, Amend B, Bedke J, Kruck S, Nagele U, Stenzl A, et al. 2013Minimally invasive percutaneous nephrolithotomy: A comparative study of The management of small and large renal stones. Urology;81:241 5.

26. Traxer O, Smith TG $3^{\text {rd }}$, Pearle MS, Corwin TS, Saboorian H, Cadeddu JA.2001Renal parenchymal injury after standard and mini percutaneous nephrostolithotomy.JUrol;165: 16935.

27. Knoll T, Wezel F, Michel MS, Honeck P, Wendt Nordahl G. 2010Do patients Benefit $t$ from miniaturized tubeless percutaneous nephrolithotomy? A comparative prospective study. J Endourol;24:1075 9. 